\title{
The Data Processing and Remote Transmission Technologies for On-Line Monitoring Of Eurytopic Distributed
}

\author{
Yu-rong Deng, Qiu-li Wu, Wei Zhang and Ze-cheng Lv \\ Guangxi Electric Power Research Institute, Nanning 530023, China \\ wu_ql.sy@gx.csg.cn
}

\begin{abstract}
Aiming at the provincial level within the grid wide-area distributed substation equipment on-line monitoring various types, processing of large amount of data and communication standard is not unified. Optimization of the structure of communication between the various levels, researched open communication format specification, established data interaction integration model. The paper studied the wide-area distributed on-line monitoring comprehensive processing unit of end stations with provincial level network of on-line monitoring system for the main platform of communication technology. Application shows that systems runs stability and meet online monitoring data collection, processing, transmission, and other functions, and has good maintainability and extensibility, adapt to various on-line monitoring in province level the development trend of the main platform for centralized management.
\end{abstract}

Keywords: monitoring system, master platform, specification, remote transmission, communication format

\section{Introduction}

In order to be safely operation and maintenance of power equipment, our country use regular Preventive test of insulation for decades. Which play an important role in the system of insulation and preventive testing method? But the long regular inspection cycle and the preventive test lacks authenticity, there exists excessive repair phenomenon, wasting a lot of manpower and material resources, resulting in unscheduled blackouts in power, decrease equipment reliability and lead to catastrophic failure in a wider range, so now have to hand in the application of the intelligent monitoring and diagnosis system for those expensive power equipment, in order to improve the operation of their value cycle, the upcoming online monitoring and fault diagnosis technology applied in power plant, substation, operation of transmission line and distributed electric power equipment, to the formation and development of the advanced system of state of repair. Condition monitoring of power equipment by using sensor technology, computer technology and signal processing technology and use it as the basis for power equipment predictive repair guarantee power system safe, reliable operation of the fundamental way, is nearly twenty years of information technology to the power set of important achievements preparation and maintenance field permeability test.

In recent years, the new sensor technology come together with the progress of signal processing technology and computer technology promoted the rapid development of online monitoring technology of power equipment. All kinds of the new sensor, including the fiber sensor, optical, magnetic and electrical optical and new type of gas sensor, widely used for on-line partial discharge monitoring of power equipment, small current and trace gas. Digital filtering, wavelet analysis technique and various pattern recognition successfully used for interference suppression in insulation online monitoring, fault identification and location. The use of computer technology, not only a single parameter 
of single power equipment monitoring, the monitoring center can also carry out condition monitoring and diagnosis based on the power system of all electric power equipment.

Numerous online monitoring system of substation equipment put into operation, can grasp the key substation equipment operating status, and helping to improve the health of the device. However, the large number of on-line monitoring systems requires higher demands on both the content of the data processing, transmission. For example Guangxi Power Grid transformer oil dissolved gas monitoring system description, the system includes more than 270 sets of monitoring devices, and these devices belong to eight manufacturers of more than 10 models. Since the communication protocol, widely distributed and functional differences, resulting information is scattered, the structure of complex situations, and increased use of difficulty, can't give full play to the role of monitoring data.

At present, the development of substation monitoring system from the initial upgrade to central distributed, and because hierarchical distributed technology, widely used in bus technology and Web technology, R \& D target changes to multi-application system[1-2]. Therefore, based on the existing research results on-line monitoring system research can be unified, standardized method; It can meet the needs of remote transmission substation monitoring data. This paper uses an embedded computer technology, communication technology of wide-area distributed, interactive data integration technology, expand the IEC61850 international standard communication norms, combined with the application of $500 \mathrm{kV}$ Jiulong substation monitoring system to study applies to communications technology to transmit data from the substation to the main station platform.

\section{System Design}

Distributed network function in accordance with the multi-stage application requirements, constructed intensive early warning system for substation equipment and diagnostic fault of Guangxi Power Grid. Based on a large collection of technical data imputation wide area distribution of various types of monitoring data, and the data is forwarded to the provincial network layer data center in accordance with the openness of the communication format. We can Use the web application Guangxi Power Grid Electrical Equipment Safety Warning Decision Platform (main station platform), remote monitoring and diagnosis of different types of device status. Online monitoring data exchange within the network, through the firewall, Intrusion-Prevention System (IPS), the Internet and other on-site monitoring and behavior control Internet isolation.

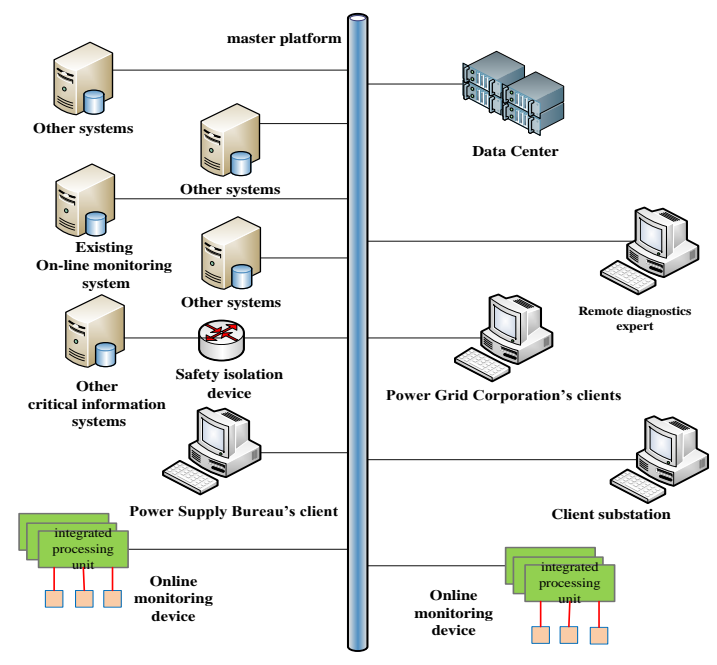

Figure 1. System Structure Diagram On-Line Monitoring Data Exchange 
In order to correctly line monitoring data to the provincial network layer, the online monitoring of integrated data processing unit (comprehensive processing unit) deployed in the substation, to achieve multi-system data aggregation and integration of multifeature measurement values, and develop an open, stable communication format for connection to the main station platform comprehensive processing unit.

Comprehensive processing unit application object is monitoring equipment, receiving and processing equipment associated with monitoring data. In order to achieve a standardized communication format for data transmission the master platform, comprehensive processing unit and the main station platform is based on standardized interfaces for interconnection, and communication design in full compliance with "smart high-voltage equipment communication technology standard" requirement. Data communication format specification follows the IEC61850 standard. That allows the device to condition data, monitoring data and other information in a unified interactive integrated modeling, making flexible and adapt to the development of sensor technology and monitoring techniques. The data communication format will support the IEC61850 specification various communication services, including Multimedia Messaging Service(MMS), Generic Object Oriented Substation Event(GOOSE),reporting, control, replace, fixed value, log, samples (Sampled Value, SV) and other services.

The main station platform set up in Guangxi Power Grid electrical equipment monitoring and early warning center, enabling analytical processing and showcase a wide area of substation equipment monitoring data. Its data exchange module with information transfer between nodes computer networks, data sharing and distributed processing and other functions, and obtain data from external systems (including data centers).

Comprehensive processing unit can communicate with the master platform, the main features include comprehensive processing unit access from inside the substation monitoring system for all types of online data, and processing; generate standard IEC61850 interactive data integration model, and MMS way to access the main station platform . This approach can be adapted to the smart grid requirements for data transmission, but also can solve the big grid monitoring different types of data, different communication protocol heterogeneous data transmission problems. A communication network structure contains the basic functions of the template is shown in Figure 1.

IEC61850 is suit to substation automation communication standard, it not only adapt to the development of communication technology, suit to complex and changeable application function of substation automation, but also be able to scientifically assess the performance of communication in substation automation system. What's more, it adapts to the distributed application of substation automation system functions to achieve the interoperability of application and combination, the true sense of the substation automation related equipment support. IEC61850 support interoperability, because it has the following characteristics, the system hierarchical structure distribution, Object Oriented Unified Modeling and data description, with abstract communication service interface and specific communication service mapping function.

In order to meet the data type and transmission capabilities of the smart grid, comprehensive processing unit must be high stability. Comprehensive processing unit must be addressed access to types of monitoring data, large data transfers, while the issue of field data collection and upload data to the master platforms; therefore chosen two gigabit Ethernet interfaces and scalable 8-channel tape optically isolated RS485 serial.

For all types of monitoring data, the time course of the overlay precision requirements, the use of the necessity and difficulty of IEC61850-9-2-point method, proposes a method to realize the value of transmission sampling point. IEC61850-9-2 and IEC61850-9-1 compared to advantage, these advantages include the ability to configure the data content and number, flexible configuration, convenient data sharing. Processing the response time history data is less than one second, which is greater than 200 for storing the logical nodes, each node contains the logical data group 10, the storage period limit of five 
minutes, the upper limit of the storage period is greater than one year. The real-time data processing response time is less than 0.1 seconds, the collection and processing of the sum of the station all the time online monitoring unit does not exceed 5 minutes.

\section{Open Communication Format Specification}

To meet the requirements of remote centralized monitoring communications technology, the future master should have an open platform, digital, multi-point communication control the underlying network. In traditional substation automation systems, communication protocols from different vendors are not unified, the impact of the openness of the station LAN communications, increasing the communication protocol conversion work, or even result in unreliable communication. To solve this problem, to adopt the communication standard in the industry (IEC61850, DL/T860, TCP / IP, etc.) based on the development of open communication format specification to be able to adapt to the current monitoring data communication needs and trends.

Comprehensive processing unit follow DL/T860 agreement to adapt the provisions of IEC61850 protocol. And in order to take into account other types of demand, the need to support the Web Service, I2, and 104 and other communication protocols. Which, Web Service can support heterogeneous systems interconnection agreement, I2 protocol is widely adopted in China to monitor data access interface protocol, 104 protocol is a traditional power system communication protocol.

Since comprehensive processing unit shall access the monitoring data types, it should have at least 6-access channel signal, but also need to support both IPv4 and IPv6 addresses assigned and conversion processing capacity. Similarly, the need for data upstream master platform shall be marked IP host and backup machines, and access to the master platform to identify and restrictions.

Access comprehensive processing unit the monitoring of transformer oil types including gas monitoring, monitoring of the mechanical properties of the circuit breaker, transformer partial discharge monitor, GIS partial discharge monitoring, switchgear partial discharge monitoring. Those monitoring system recorded data using the reference industry "standards-based DL/T860 substation equipment line monitoring device application specification" is defined, recorded data by DL/T860 Statute also requires transfer files. These systems use the recommended international standard IEC-defined data encoded files, and through COMTRADE format type XML configuration file to achieve. If the interrupt comprehensive processing unit Communication with the master platform, should restore communication automatically calls the blackout period the latest data and statistical information of all data loss. In order to meet the on-site maintenance and verification of information needs, Comprehensive processing unit provided by the current call and a remote call function to achieve historical data extraction device.

Comprehensive processing unit and master platform real-time communications capabilities, time synchronization accuracy for a comprehensive analysis of the status of the device is very important. So comprehensive processing unit and the main station platform uses Simple Network Time Protocol (SNTP) time synchronization. Among them, the master platform clock as the clock source standard, comprehensive processing unit timing calibration standard clock, and each time period for the monitoring system can be adjusted. On the time interval between one minute to twenty-four hours; and usually set to one hour.

\section{Build Interactive Data Integration Model}

Realize on-line monitoring data communication standardization and unification of integration architecture [4-5], end stations integrated unified access platform, the main processing unit data can meet the demand of the development of the integration of on-line monitoring information integration [6], on the basis of the implementation for wide-area 
distributed condition warning and diagnosis equipment. For analysis to determine equipment status data requirements, unified by the on-line monitoring substation integrated processing units with on-site monitoring data interaction, effectively integrate and share resources of all kinds of data, and then uploaded to the host platform. So you need to build a data integration model and its key technologies as follows [7].

1) Access to various types of communication standard on-line monitoring device, and established the model according to IEC61850 standard.

2) Transmission of data by way of IEC61850 or transparent manner forwarded to the master platform, and support for remote maintenance monitoring device.

3) Compliant with IEC61850 standard Web services interfaces and access interfaces and comprehensive processing unit and data server to interact.

To meet these requirements, Comprehensive processing unit and the main station platform interactive data-oriented integration model is modeling the object, and defines the data model for client / server architecture based on. The model includes system configuration, logical nodes and service type 3 parts. Details are as follows:

\subsection{System Configuration}

System configuration process compliance "DL/T860 series of standards implementation of the project technical specifications", and also contains the following details:

(1) configured in accordance with the scale of the project vision Instantiated Data Object (IDO), IED Capability Description (ICD) should contain Chinese-based string parameter description and description attributes.

(2) The Substation Configuration Description (SCD) generated automatically Configured IED Description (CID) downloaded to the monitoring device.

(3) ICD model of self-describing file needs to contain information such as the version of the modified information, modify the time described, and a modified version of the serial number.

\subsection{System Configuration}

Logical node type definition directly related to the Comprehensive processing unit Data integration of various online monitoring units within the substation. Each comprehensive processing unit are equipped with two basic logical nodes, which are used to implement logic node information management class interaction, and a description of the physical characteristics of their physical logical node class[8-9]. For $500 \mathrm{kV}$ Jiulong substation monitoring system as an example, integrated access to multiple processing units, respectively, for each main line monitoring unit transformers, circuit breakers, etc., need to configure different types of detection and control nodes. Logical nodes belonging to the model defined in Table 1 . 


\section{Table 1. Interactive Data Integration Model Logic Node Definition}

\begin{tabular}{|c|c|c|}
\hline $\begin{array}{l}\text { Functio } \\
\text { n Type }\end{array}$ & Logical node & $\begin{array}{l}\text { Logic } \\
\text { Node } \\
\text { Type }\end{array}$ \\
\hline \multirow{2}{*}{$\begin{array}{c}\text { The } \\
\text { basic logic } \\
\text { node }\end{array}$} & Management logic nodes & LLNO \\
\hline & the physical logical node & LPHD \\
\hline \multirow{8}{*}{$\begin{array}{l}\text { Transfor } \\
\text { mer }\end{array}$} & Master IED logical nodes & SPTR \\
\hline & the oil gas logical nodes & SIML \\
\hline & $\begin{array}{c}\text { logical nodes transformer } \\
\text { PD }\end{array}$ & SPDC \\
\hline & $\begin{array}{l}\text { Transformer bushing } \\
\text { insulation logical nodes }\end{array}$ & ZBSH \\
\hline & $\begin{array}{l}\text { logical nodes measuring } \\
\text { conditions }\end{array}$ & GGIO \\
\hline & air control logic nodes & GGIO \\
\hline & OLTC logical node & SLTC \\
\hline & $\begin{array}{l}\text { non-power protection logic } \\
\text { node }\end{array}$ & GGIO \\
\hline \multirow{5}{*}{$\begin{array}{l}\text { the } \\
\text { circuit } \\
\text { breaker }\end{array}$} & Master IED logical nodes & GGIO \\
\hline & $\begin{array}{l}\text { the circuit breaker } \\
\text { monitoring logic node }\end{array}$ & SCBR \\
\hline & $\begin{array}{c}\text { the node logic circuit } \\
\text { breaker operating mechanism }\end{array}$ & SOPM \\
\hline & $\begin{array}{l}\text { isolation switch control } \\
\text { logic node } \\
\end{array}$ & CSWI \\
\hline & $\begin{array}{l}\text { SF6 gas monitoring logic } \\
\text { node }\end{array}$ & SIMG \\
\hline \multirow{2}{*}{ Other } & Environmental monitoring, & MMET \\
\hline & temperature load & STMP \\
\hline
\end{tabular}

\subsection{Functional Service}

State the comprehensive processing unit external communications, including "connected," "abort" and "release", which are suitable for different mission requirements. "Comprehensive processing unit" to establish a connection with the client number is greater than 16, found that communication stop in less than one minute. Similarly, the client found the server stops running time of less than two minutes [10]. Therefore, the communication of each client configuration process will be in accordance with established requirements. Meanwhile, although the logical node model to achieve a standardized communication formats, but in order to ensure the quality of communication, but also to improve its functional services.

1) Data access service in accordance with the index structure organization. It can be read in the directory include: server directory, the directory logical device, logical node directory, data directory.

Parameter settings for specific content types include: setting data set, the combined data set, the data directory.

Operations on data types include: call values, call the value of the data collection, the call data definitions.

All of the above reading services, typically for each specific operation and is accompanied by a corresponding object of several services. However, calling the directory, the definition reads data, call data values are required for data and control module can support simultaneously. Conversely, setting the data value is only allowed for those operational data, such as control modules, remote controlled, modify the set point, substituted data. 
2) Adoption of the report command to achieve "comprehensive processing unit" for the "master platform" reporting services, involving functional modules include: the report calls cache control module, set the cache control module report, the report calls a noncached control module, set non-cached report control module. Data services will be integrated in the report ICD file, and can be change by the SCD file, the data collection is not required to dynamically create and modify. Because Reporting Services is to achieve the client's call, and to meet real-time requirements of different clients, client reporting services also support online reporting service parameter settings.

3)The type of document services include: call file, call the file attributes. Files service parameter settings will be performed in accordance with the provisions DL/T860.81.According to the needs of the client, the file name of the file services can not be empty, and the file text data type octet string. In addition, in order to avoid conflicts, when a client reads the file directory, select all the way does not allow ,can use "**.*”to read the parameter.

4) Log service function is to transmit historical information on-line monitoring, the main storage for each Intelligent Electronic Device (IED) information on the history of operating conditions. In order to ensure data consistency, a single service, only one log control module, and log storage media entities using a relational database.

5) Remote self test service can be a single point of control, test items include: sensor wiring status, IED and comprehensive processing unit connection status, fieldbus connection status, IED system status, and other content. Detection can be achieved through the automatic mode or manual mode.

Based interactive design data integration model, applied research communications specifications, preparation of technical specifications online monitoring communications within the applicable provincial power grid, Such as "substation equipment online monitoring data transmission protocol", "substation equipment on-line monitoring spectral data format specification" and will regulate online monitoring communications technology foundation.

\section{Conclusion}

Online monitoring technology for the insulation of electrical equipment with real-time continuous, which directly and accurately reflect the real situation in the operating conditions of equipment, the insulation test is simple, rapid, do not affect the normal operation of power system, easy to develop into the monitoring and diagnosis system for intelligent, networked computer system benefits and power systems, incorporated into the automated system of the electric power system, and has broad application prospects.

The comprehensive processing unit and main transformer online monitoring platform of communication technology meet the requirement of monitoring technology status and development trend, combined with the international standard specification of communication design. Data integration model, developed by specification for each function block and concrete structure, can summarize various types of monitoring data fusion, make the interchangeable between different monitoring devices, monitoring system structure with high dispersion and advantages of flexibility. Monitoring device and the main platform of data communication format specification, although in accordance with international standards, with openness, due to the development of the monitoring communication without the restriction of unified standard, caused the regions and units communication format specification and a wide variety of situation, therefore, need careful analysis based on engineering practice, the reasonable selection. 


\section{References}

[1] W. Nan, C. Zhi-ye, L. Fang-cheng, "A survey of on-line monitoring and diagnosis for capacitive equipment", Power System Technology, vol. 27, no. 8, (2003), pp. 72-76.

[2] Z. Yi-xin and Q. Jun, "Discussion on integrated power data network", Telecommunications for Electric Power System, vol. 31, no. 11, (2010), pp. 28-30.

[3] L. Ze-you and C. Yun-qing, "Experience of using IEC 60870-5-103 and IEC 60870-5-104 transmission protocols", Automation of Electric Power Systems, vol. 27, no. 4, (2003), pp. 1-3.

[4] X. Bing-yuan, "Power grid enterprise information integration based on SOA platform of research and practice", Electric Power Information, vol. 6, no. 1, (2008), pp. 70-75.

[5] W. Yongfu, "Research and practice to electric power enterprise information integration", Electric power informatization, vol. 4, no. 6, (2006), pp. 5-88.

[6] Z. Chun-Jie, "Power integrated information integration strategy analysis and practice", Electric power informatization, vol. 5, no. 6, (2007), pp. 34-37.

[7] Z. Jin-jiang, G. Chuang-xin, C. Yi-jia, "Substation equipment condition monitoring system and IEC model coordination", Automation of Electric Power Systems, vol. 33, no. 20, (2009), pp. 67-72.

[8] C. Yonghong, C. Yu and M. Yongpeng, "Development of insulation online monitoring system of transformer substation integrated power device", High voltage technology, vol. 33, no. 8, (2007), pp. 6165.

[9] L. Guoqing, Z. Zhong and W. Zhenhao, "The on-line monitoring of dielectric loss of capacitive apparatus", Grid technology, vol. 31, no. 7, (2007), pp. 55-58.

[10] C. Haibo, W. Cheng and L. Junfeng, "Application of UHV transmission line on-line monitoring technology" Grid technology, vol. 33, no. 10, (2009), pp. 67-71.

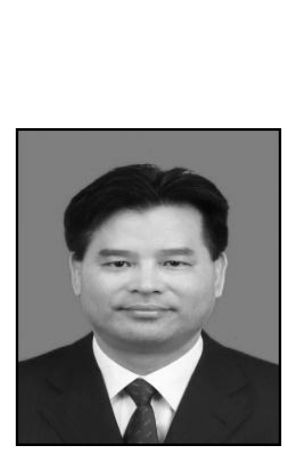

\section{Authors}

Y. R. Deng, Be engaged in On-line monitoring of electric equipment of radio frequency identification technology, Excellence at warning center maintenance management, condition evaluation, and reliability analysis. 